\title{
Necessidade de tratamento odontológico autopercebida e normativa em indivíduos de três distritos sanitários de Porto Alegre/RS
}

\author{
Self-perceived and normative need for dental treatment of individuals \\ from three health districts of Porto Alegre, RS, Brazil
}

Maiara Mundstock Jahnke*

Monique Estér Ponte*

Claídes Abegg ${ }^{* * *}$

Victor Nascimento Fontanive ${ }^{* * * *}$

Rosane Davoglio ${ }^{* * * *}$

\section{Resumo}

Objetivo: este estudo teve como objetivo verificar o nível de concordância entre necessidade normativa e autopercebida de tratamento odontológico em indivíduos de 50 a 74 anos e analisar a associação entre variáveis sociodemográficas e necessidade de tratamento normativa e autopercebida. Sujeitos e método: este é um estudo transversal de base populacional realizado em três distritos sanitários de Porto Alegre/RS. A amostra foi de múltiplos estágios; os dados foram coletados por examinadores e anotadores devidamente calibrados nos domicílios do entrevistado, depois de assinado o termo de consentimento livre e esclarecido. Os dados foram analisados por intermédio do SPSS 16.0. Resultados: foram entrevistados e examinados 720 indivíduos, dos quais $57,7 \%$ eram mulheres e 42,3\%, homens. Dos entrevistados, 582 $(80,8 \%)$ relataram necessitar de tratamento odontológico e $490(68,1 \%)$ apresentavam necessidade normativa de tratamento. Observou-se um Kappa de 0,86 no nível de concordância entre necessidade autopercebida e normativa. Conclusão: a necessidade de tratamento autopercebida esteve relacionada ao sexo. Os homens relataram ter mais necessidade de tratamento do que as mulheres. Foi possível observar alta concordância entre necessidade de tratamento autopercebida e normativa. Não se observou associação entre necessidade de tratamento normativa e variáveis socioeconômicas.

Palavras-chave: Autopercepção. Necessidade de tratamento. Saúde bucal.

\section{Introdução}

Nos últimos anos, tem-se dado destaque ao enfoque social para medir a saúde bucal da população e à tomada de decisão sobre a necessidade de tratamento, levando-se em consideração a necessidade percebida pelos indivíduos. Esta pode ser definida como a necessidade considerada importante baseada em sentimentos subjetivos daquilo que os indivíduos realmente querem ${ }^{1}$.

Cirurgiã-dentista, residente em Saúde da Família e Comunidade, Programa de Residência Integrada em Saúde/GHC. Unidade de Saúde Vila Floresta, Porto Alegre, Rio Grande do Sul, Brasil.

Cirurgiã-dentista, aluna do curso de Especialização em Cirurgia e Traumatologia Bucomaxilofacial da Pontifícia Universidade Católica do Rio Grande do Sul. Porto Alegre, Rio Grande do Sul, Brasil.

Cirurgiã-dentista, doutora em Odontologia, professora associada do Departamento de Odontologia Preventiva e Social, Faculdade de Odontologia, Universidade Federal do Rio Grande do Sul. Porto Alegre, Rio Grande do Sul, Brasil.

**** Cirurgião-dentista, mestre em Odontologia, Grupo Hospitalar Conceição, Serviço de Saúde Comunitária. Porto Alegre, Rio Grande do Sul, Brasil. Cirurgiã-dentista, doutora em Odontologia, professora adjunta da Universidade Federal do Vale do São Francisco. Petrolina, Pernambuco, Brasil. 
As medidas mais utilizadas em odontologia para medir o nível de saúde bucal da população são, em geral, o Índice de Dentes Cariados, Perdidos e Obturados (CPOD) para a cárie e o Índice Comunitário de Necessidades de Tratamento Periodontal (CPITN) para a doença periodontal. Embora aplicadas em inúmeros estudos, as limitações desses índices têm sido objeto de várias discussões ${ }^{2}$. Uma das limitações do CPOD e do CPITN é que estes não levam em consideração a percepção subjetiva em relação à saúde bucal, isto é, pouco consideram a maneira como a saúde bucal afeta a vida diária dos sujeitos. Assim, existe a necessidade da inclusão de outros indicadores, além dos clínicos, para avaliar as condições e necessidades de saúde da populaçãooe-5.

A definição dessa necessidade tem fundamental importância ao se planejar a assistência à saúde. A necessidade de atenção à saúde, usualmente, é definida por parâmetros normativos - com base na avaliação clínica realizada pelos profissionais. Entretanto, outros parâmetros também são necessários, na medida em que o entendimento do significado de saúde e doença tornou-se passível de interpretações mais amplas do que era anteriormente ${ }^{6}$. Atualmente, a saúde é vista não somente como ausência de doença, mas como resultante das experiências sociais dos sujeitos, estando estreitamente vinculada à sua qualidade de vida ${ }^{7}$.

A necessidade normativa é definida pela quantidade de serviços odontológicos avaliados por cirurgiões-dentistas que determinam o tratamento a ser realizado, objetivando a prevenção da doença e o restabelecimento da saúde, baseado no conhecimento existente ${ }^{6}$. A necessidade percebida, por sua vez, é pessoal e subjetiva, resultante de crenças, conhecimento e julgamento de valores da pessoa afetada, sendo influenciada por fatores psicológicos, socioeconômicos e culturais ${ }^{6}$.

Desse modo, discute-se que, na atualidade, o planejamento das ações em saúde deve ir além da avaliação da necessidade normativa, incluindo aquela percebida pelos sujeitos. Logo, tem-se a oportunidade de montar um quadro claro de objetivos a serem trabalhados, o que nos permite determinar metas e prioridades ${ }^{6}$.

Os objetivos deste estudo foram verificar o nível de concordância entre necessidade normativa e autopercebida de tratamento odontológico em indivíduos de 50 a 74 anos e analisar a associação entre variáveis sociodemográficas e necessidade de tratamento normativa e autopercebida.

\section{Sujeitos e método}

Este trabalho faz parte de um estudo maior que foi realizado na cidade de Porto Alegre, Rio Grande do Sul, Brasil, entre os anos de 2006 e 2010, quando foi efetuada a coleta de dados. Trata-se de um estudo analítico observacional, do tipo transversal, de base populacional. A cidade onde se realizou a pesquisa possui 82 bairros e está dividida em 16 distritos sanitários, dos quais três participaram da pesquisa - Partenon, Leste e Glória. A população alvo compreendeu indivíduos desses três distritos sanitários. A faixa etária entrevistada foi entre 50 e 74 anos.

Por se tratar de uma investigação que compreendia múltiplos objetivos e por não se conhecer a prevalência do desfecho, o cálculo amostral foi realizado projetando-se uma prevalência para o impacto odontológico de 0,50, um intervalo de confiança de 95\% (IC95\%) e margem de erro bilateral de $4 \%$. Obteve-se uma amostra de 601 sujeitos. Considerando-se a possibilidade de perdas, calculou-se um acréscimo de cerca de $10 \%$, chegando-se a 661 indivíduos. Acrescentou-se um efeito de delineamento de $20 \%$, obtendo-se uma amostragem final correspondente a 793 sujeitos $^{8}$. Esse tamanho de amostra tem o poder de $80 \%$ para as diferenças na necessidade autopercebida de tratamento entre os sexos obtidas no presente estudo.

Foi realizada uma amostragem aleatória de múltiplos estágios. No primeiro estágio, dentre os 16 distritos sanitários de Porto Alegre, foram sorteados três. O número de domicílios visitados em cada distrito foi proporcional ao total de domicílios existentes. No segundo estágio, foram amostrados os setores censitários, utilizando amostragem aleatória. Em cada setor, foram sorteados um quarteirão e uma esquina. A partir da esquina sorteada, foram iniciadas as visitas a todos os domicílios, até se completar o número necessário da amostra daquele setor.

A coleta de dados foi realizada por intermédio de um questionário estruturado, composto por questões que objetivaram conhecer a situação socioeconômica dos sujeitos e necessidade autopercebida de tratamento e do exame clínico. As questões referentes aos dados socioeconômicos e necessidade autopercebida de tratamento foram adaptadas do estudo nacional SB-Brasil 2003. O questionário foi testado em estudo piloto e utilizado em estudo com a mesma população por Fontanive et al..$^{9}$ (2013).

A variável necessidade de tratamento normativa foi obtida por meio da combinação do componente cariado do CPOD - dente cariado (sim ou não) - e dos dados de necessidade de prótese superior e inferior (sim ou não) coletados pelo exame clínico. A necessidade autopercebida de tratamento foi avaliada com a questão: você considera que necessita de tratamento atualmente? (sim ou não).

As variáveis sociodemográficas idade (50 a 59 anos e 60 a 74 anos), sexo (feminino e masculino), renda (até 415 reais, 416 reais ou mais) e escolaridade ( 0 a 5 anos de estudo e 6 anos de estudo ou mais) foram utilizadas em conjunto com a informa- 
ção da necessidade autopercebida de tratamento odontológico e necessidade normativa. A variável renda foi coletada de forma contínua e, posteriormente, dicotomizada com base no salário mínimo vigente na ocasião do estudo. A escolaridade foi considerada pelos anos completos de estudo e, posteriormente, dicotomizada com base na distribuição da frequência ${ }^{9}$.

A coleta de dados foi efetuada nos domicílios por quatro duplas compostas por um examinador (cirurgião-dentista) e um anotador (estagiário) devidamente calibrados entre os meses de maio de 2008 e março de 2009. A reprodutibilidade intra e interexaminador de todos os índices bucais ficou acima de 0,81 e 0,76 , respectivamente, no início da pesquisa. Durante a pesquisa, foram realizados entrevistas e exames clínicos, sob luz artificial, utilizando espelho plano e sonda periodontal nos padrões da Organização Mundial da Saúde (OMS). Para o exame clínico, foram utilizados os critérios da OMS complementados por critérios do Levantamento SB Brasil $2003^{10}$.

O controle de qualidade foi realizado a partir da revisão das fichas pelo anotador após o término da entrevista, pela digitação dos dados por participantes treinados, pelo método da dupla digitação no Epi info 6.0 e pela identificação de missings e outliers no programa SPSS 16.0.

Inicialmente, realizou-se análise de distribuição de frequência das variáveis do estudo. Para verificar a concordância entre necessidade normativa e necessidade autopercebida, utilizou-se o coeficiente de concordância de Kappa. A associação entre as variáveis sociodemográficas e necessidade normativa e autopercebida de tratamento foi realizada mediante o cálculo de qui-quadrado de Pearson. O nível de significância adotado foi de $\mathrm{p}<0,05$. Todas as análises foram realizadas com o programa SPSS 16.0.

Este trabalho faz parte da pesquisa "Avaliação do impacto odontológico no desempenho diário dos indivíduos de 50 a 74 anos em Porto Alegre/ RS", aprovada pelo Comitê de Ética em Pesquisa da Faculdade de Odontologia da UFRGS no dia $24 / 01 / 2006$, ata número 01/06, com número do processo 46/05.

Antes do início das entrevistas, cada participante recebeu explicações sobre a realização da pesquisa, cujos objetivos estão explicitados no Termo de Consentimento Livre e Esclarecido, que cada participante assinou.

\section{Resulltados}

Foram entrevistados e examinados 720 indivíduos, perfazendo uma taxa de resposta de $90,8 \%$. As perdas ocorreram por recusa dos indivíduos em participar da entrevista e por não presença do indivíduo no domicílio após três tentativas de contato. Dos respondentes, $57,7 \%$ eram mulheres e $42,3 \%$, homens; 371 (51,5\%) eram do distrito sanitário Partenon; 241 (33,5\%), do distrito Leste; e 108 (15\%), do distrito Glória, em Porto Alegre. Tinham entre 50 e 59 anos $(53,4 \%)$ e entre 60 e 74 anos $(46,6 \%)$. Possuíam renda pessoal de um salário mínimo ou mais $(68,5 \%)$. A escolaridade dos entrevistados era de seis anos ou mais para $477(62,1 \%)$ indivíduos.

Dentre os participantes do estudo, $582(80,8 \%)$ disseram que necessitavam de tratamento odontológico. Em relação à necessidade normativa de tratamento, $490(68,1 \%)$ necessitavam de tratamento a partir do exame clínico. No que diz respeito ao nível de concordância entre a necessidade autopercebida e normativa, observou-se um Kappa de 0,86, demonstrando alta concordância entre as duas medidas ${ }^{11}$.

O CPO-D foi menor que 25 em 395 (54,9\%) indivíduos. A média desse índice foi de 23,3, com desvio padrão de 9,7. No momento do exame clínico, 474 $(65,9 \%)$ indivíduos não possuíam lesões de cárie ativas, $431(56,5 \%)$ não necessitavam de prótese superior e apenas $242(31,9 \%)$ não necessitavam de prótese inferior.

A Tabela 1 mostra que não foi observada associação estatisticamente significativa entre necessidade de tratamento autopercebida e as variáveis socioeconômicas idade, escolaridade e renda. No que diz respeito à distribuição entre os sexos, foi verificado que os homens relataram maior necessidade autopercebida do que as mulheres, com 74,3\% e $63,7 \%$ respectivamente. Essas duas variáveis possuem associação estatística, com $p=0,003$.

A variável necessidade de tratamento normativa não esteve associada às variáveis socioeconômicas (idade, sexo, escolaridade, renda), conforme Tabela 2 
Tabela 1 - Necessidade autopercebida de tratamento odontológico relacionada às variáveis sociodemográficas. Porto Alegre, 2012

\begin{tabular}{|c|c|c|c|c|c|c|}
\hline \multirow{2}{*}{ Variáveis } & \multirow{2}{*}{$\mathrm{N}$} & \multicolumn{2}{|c|}{ Sim } & \multicolumn{2}{|c|}{ Não } & \multirow{2}{*}{ Valor de $p$} \\
\hline & & $\mathrm{n}$ & $\%$ & $\mathrm{n}$ & $\%$ & \\
\hline \multicolumn{7}{|l|}{ Sexo } \\
\hline Masculino & 303 & 225 & 74,3 & 78 & 21,7 & 0,003 \\
\hline Feminino & 416 & 265 & 63,7 & 151 & 36,3 & \\
\hline \multicolumn{7}{|l|}{ Idade } \\
\hline 50-59 anos & 382 & 267 & 69,9 & 115 & 30,1 & 0,285 \\
\hline $60-74$ anos & 337 & 223 & 66,2 & 114 & 33,8 & \\
\hline \multicolumn{7}{|l|}{ Escolaridade } \\
\hline$\leq 5$ anos & 273 & 184 & 67,4 & 89 & 32,6 & 0,720 \\
\hline$\geq 6$ anos & 447 & 307 & 68,7 & 140 & 61,1 & \\
\hline Renda & & & & & & \\
\hline$\leq 1$ Salário mínimo & 230 & 150 & 65,2 & 80 & 34,8 & 0,256 \\
\hline > 1 Salário mínimo & 491 & 341 & 69,5 & 150 & 30,5 & \\
\hline
\end{tabular}

Tabela 2 - Necessidade normativa de tratamento odontológico relacionada às variáveis sociodemográficas. Porto Alegre, 2012

\begin{tabular}{|c|c|c|c|c|c|c|}
\hline \multirow{2}{*}{ Variáveis } & \multirow{2}{*}{ N } & \multicolumn{2}{|c|}{ Sim } & \multicolumn{2}{|c|}{ Não } & \multirow{2}{*}{ Valor de $p$} \\
\hline & & $\mathrm{n}$ & $\%$ & $\mathrm{n}$ & $\%$ & \\
\hline \multicolumn{7}{|l|}{ Sexo } \\
\hline Masculino & 303 & 246 & 81,2 & 57 & 18,8 & 0,825 \\
\hline Feminino & 416 & 335 & 80,5 & 81 & 19,5 & \\
\hline \multicolumn{7}{|l|}{ Idade } \\
\hline 50-59 anos & 382 & 304 & 79,6 & 78 & 20,4 & 0,374 \\
\hline $60-74$ anos & 337 & 277 & 82,2 & 60 & 17,8 & \\
\hline \multicolumn{7}{|l|}{ Escolaridade } \\
\hline$\leq 5$ anos & 273 & 215 & 78,8 & 58 & 21,2 & 0,268 \\
\hline$\geq 6$ anos & 447 & 367 & 82,1 & 80 & 17,9 & \\
\hline Renda & & & & & & \\
\hline$\leq 1$ Salário mínimo & 230 & 184 & 80 & 46 & 20 & 0,737 \\
\hline > 1 Salário mínimo & 491 & 398 & 81,1 & 93 & 18,9 & \\
\hline
\end{tabular}




\section{Discussão}

Este é um dos primeiros estudos de base populacional, realizado em população brasileira na faixa etária de 50 a 74 anos, investigando a relação entre necessidade normativa e autopercebida de tratamento odontológico. Os resultados demonstraram uma alta concordância entre a necessidade percebida pelos sujeitos e aquela avaliada pelos profissionais.

Diferentemente dos resultados encontrados no presente estudo, investigações desenvolvidas com populações de outros países constataram uma pobre relação entre os indicadores subjetivos de saúde oral e os indicadores clínicos, na medida em que a opinião dos pacientes sobre saúde e necessidade de tratamento oral raramente ou até mesmo nunca coincidia com a opinião do clínico ${ }^{12}$. Um único estudo brasileiro encontrado na literatura observou associação estatística entre autopercepção de necessidade de tratamento e necessidade de tratamento por cárie e necessidade de prótese em uma ou duas arcadas ${ }^{13}$.

A autopercepção da necessidade de tratamento pode ser considerada como uma combinação do impacto que a doença tem sobre os sujeitos, com as percepções e atitudes destes em relação à mesma doença. Ela é subjetiva, variando de indivíduo para indivíduo, dependendo do contexto histórico e socioeconômico no qual ele está inserido ${ }^{13}$.

É possível que o nível de concordância encontrado no presente estudo esteja relacionado a uma necessidade reprimida de atenção odontológica. Em estudo com a mesma população, foi observado que $43,5 \%$ dos indivíduos apresentaram necessidade de reabilitação protética do arco superior; $68,1 \%$, indicação de reabilitação protética no arco inferior; e $34,1 \%$, lesão de cárie ativa ${ }^{14}$. Dados de estudos anteriores mostram a falta de atenção a que a população brasileira dessa faixa etária está sujeita ao longo da história ${ }^{15}$.

Em Porto Alegre, observa-se uma carência de centros de especialidades odontológicas de prótese dentária, dificultando a reabilitação protética de que a população dessa cidade necessita, comprometendo o princípio da integralidade proposto pelo Sistema Único de Saúde ${ }^{14}$.

Em relação à necessidade normativa medida a partir das variáveis dente cariado, necessidade de prótese superior e necessidade de prótese inferior, observou-se que $68,1 \%$ necessitavam de alguma atenção. Segundo levantamento epidemiológico realizado em 2003, dos indivíduos entre 65 e 74 anos da região Sul, 46,32\% necessitam de prótese inferior e $19,55 \%$, de prótese superior ${ }^{10}$. Crispim, Saupe e Boing encontraram valores ainda maiores de necessidade de prótese em idosos de Santa Catarina $(63,3 \%$ para prótese superior e $82,1 \%$ para prótese inferior $)^{16}$.
No presente estudo, encontrou-se necessidade autopercebida de tratamento em $80 \%$ da população investigada. Já no levantamento nacional das condições de saúde bucal da população brasileira, que investigou a necessidade de tratamento subjetiva em idosos (65 a 74 anos), $55 \%$ a percebiam ${ }^{10}$. Em investigação realizada por Moreira, foi observada diferença na autopercepção de tratamento odontológico entre idosos edêntulos e não edêntulos, além de associação entre idade e menor necessidade subjetiva ${ }^{17}$. É possível que neste estudo a necessidade de tratamento autopercebida tenha maior prevalência por fazerem parte da amostra indivíduos a partir de 50 anos. Entretanto, não se observou associação estatística entre as variáveis idade e necessidade autopercebida de tratamento. Além disso, em estudo com a mesma população, observou-se que os indivíduos apresentavam alto impacto no desempenho das atividades diárias causado por problemas odontológicos como perda dentária e próteses mal adaptadas. Essas questões podem estar influenciando a necessidade autopercebida de tratamento ${ }^{18}$.

Chama a atenção o fato de que os homens relatam ter mais necessidade de tratamento do que as mulheres. No entanto, dados sobre procura por serviços odontológicos na mesma população não mostraram diferença estatisticamente significativa para a variável sexo ${ }^{19}$. Sendo assim, pode-se concluir que os homens relatam mais necessidade de tratamento que as mulheres, mas não há uma maior procura por serviços de atendimento odontológico por esse sexo. Mesmo percebendo necessidade de tratamento odontológico, é provável que, por questões socioculturais, os homens não busquem atenção odontológica.

A adequação do desenho do estudo, a calibração dos examinadores, o treinamento dos anotadores e a técnica de dupla digitação usada para o controle de qualidade dos dados conferem validade aos resultados obtidos. Entretanto, o estudo apresenta algumas limitações: a homogeneidade da amostra em relação à escolaridade e renda pode ter interferido no resultado, que não permitiu encontrar diferenças entre os grupos. Além disso, os achados não podem ser extrapolados para toda a população de Porto Alegre.

\section{Conclusões}

Tendo em vista os objetivos propostos pelo presente trabalho, no grupo de indivíduos entrevistados e examinados, pode-se concluir que a necessidade normativa e a necessidade autopercebida de tratamento odontológico possuem um alto nível de concordância.

A necessidade de tratamento autopercebida está relacionada ao sexo, em que os homens relatam ter mais necessidade de tratamento do que as 
mulheres, não estando associada à idade, renda e escolaridade. Não se observou associação entre a necessidade de tratamento normativa e as variáveis socioeconômicas investigadas.

\section{Abstract}

Objective: this study aimed to assess the level of agreement between normative and self-perceived need for dental treatment of individuals aged 50 to 74 years, as well as to analyze the association between sociodemographic variables, and normative and self-perceived need for treatment. Subjects and methods: this study is a population-based cross-sectional study performed in three health districts of Porto Alegre, RS, Brazil. A random multi-stage sampling was used. Data were collected in the homes of the respondents by properly calibrated examiners and annotators after the respondents had signed the informed consent form. Data were analyzed with the use of the SPSS 16.0. Results: 720 individuals were interviewed and examined. From them, $57.7 \%$ were women, and $42.3 \%$ were men. From the respondents, $582(80.8 \%)$ said they needed dental treatment, and $490(68.1 \%)$ presented normative need for treatment. A 0.86 Kappa was observed in the level of agreement between self-perceived need and normative need. Conclusions: self-perceived need for treatment was associated to gender. Men reported having more need for treatment than women. A high concordance was observed between self-perceived and normative need for treatment. No association was found between socioeconomic variables and normative need for treatment.

Keywords: Self-perception. Need for treatment. Oral Health.

\section{Referências}

1. Daly B, Watt R, Batchelor P, Treasure E. Introduction to the principles of public health. In: Daly B, Watt R, Batchelor P, Treasure E. Essential Dental Public Health. Oxford: Oxford University Press; 2006. p. 15.

2. Locker D. An introduction to behavioral science \& dentistry. New York/London: Routledge; 1989.

3. Cushing AM, Sheiham A, Maizels J. Developing socio-dental indicators - the social impact of dental disease. Comm Dent Health 1986; 3(1):3-17.

4. Maizels J, Maizels A, Sheiham A. Dental disease and health behaviour: the development of an interactional model. Comm Dent Health and Dental Pract 1991; 8(4):311-21.

5. Sheiham A. A determinação de necessidades de tratamento odontológico: uma abordagem social. In: Pinto VG. Saúde bucal coletiva. São Paulo: Santos; 2000. p. 223-50.

6. Sheiham A, Tsakos G. Avaliando necessidades através da abordagem sócio-odontológica. In: Pinto VG. Saúde bucal coletiva. São Paulo: Santos; 2008. p. 287-316.

7. Dickson M, Abegg C. Desafios e oportunidades para a promoção de saúde bucal. In: Buischi YP. Promoção de saúde bucal na clínica odontológica. São Paulo: Artes Médicas; 2000. p. 39-71.
8. Fontanive VN. A relação entre variáveis clínicas de saúde bucal e qualidade de vida dos indivíduos entre 50 e 74 anos de três distritos sanitários de Porto Alegre [Dissertação de Mestrado]. Porto Alegre: Faculdade de Odontologia da Universidade Federal do Rio Grande do Sul; 2010.

9. Fontanive VN, Abegg C, Tsakos G, Oliveira M. The association between clinical oral health and general quality of life: a population-based study of individuals aged 50-74 in Southern Brazil. Comm Dent Oral Epidemiol 2013; 41(2):15462 .

10. BRASIL. Ministério da Saúde. Projeto SB Brasil 2003: condições de saúde bucal da população brasileira 2002-2003, resultados principais. Brasília: Ministério da Saúde; 2004. [citado 2011 Nov 10]. Disponível em URL: http://dab.saude. gov.br/cnsb/publicacoes.php.

11. Landis JR, Koch GG. The measurement of observer agreement for categorical data. Biometrics 1977; 33:159-74.

12. Seremidi K, Koletsi-Kounari H, Kandilorou H. Self-reported and clinically-diagnosed dental needs: determining the factors that affect subjective assessment. Oral Health Prev Dent 2009; 7(2):183-90.

13. Martins AMEBL, Barreto SM, Pordeus IA. Fatores relacionados à autopercepção da necessidade de tratamento odontológico entre idosos. Rev Saúde Publ 2008; 42(3):487-96.

14. Mallmann FH, Toassi RFC, Abbeg C. Perfil epidemiológico do uso e necessidade de prótese em indivíduos de 50-74 anos em Porto Alegre, Rio Grande do Sul, 2008. Epidemiol Serv de Saúde 2012, 21(1):79-88.

15. Moreira RS, Nico LS, Tomita NE, Ruiz T. A saúde bucal do idoso brasileiro: revisão sistemática sobre o quadro epidemiológico e acesso aos serviços de saúde bucal. Cad Saúde Públ 2005; 21(6):1665-75.

16. Crispim AJ, Saupe R, Boing F. Perfil epidemiológico do uso e necessidade de prótese e de alterações de tecidos moles bucais em idosos de uma comunidade de Itajaí - SC. Arq Catarinenses Med 2009; 38(2):53-7.

17. Moreira RS, Nico LS, Sousa MLR. Fatores associados à necessidade subjetiva de tratamento odontológico em idosos brasileiros. Cad Saúde Publ 2009; 25(12):2661-71.

18. Bittencourt V, Abegg C, Fontanive VN. O impacto da saúde bucal nas atividades diárias de indivíduos de 50 a 74 anos em três distritos sanitários de Porto Alegre/RS. Rev Fac Odontol Univ Passo Fundo. 2013; 18(1):37-43.

19. Jahnke MM, Abegg C. Necessidade de tratamento auto-percebida e a utilização dos serviços odontológicos de indivíduos em Porto Alegre - RS. In: $22^{\circ}$ Salão de Iniciação Científica: 2010; Porto Alegre. Anais. Porto Alegre: Ed. da UFRGS/PROPESQ; 2010. Resumo 007.

Endereço para correspondência:

Maiara Mundstock Jahnke

Rua Comendador Albino Cunha 53, apto. 302,

Cristo Redentor

91040-040 Porto Alegre/RS

Fone: (51) 9752-7967

E-mail: maiarajahnke@yahoo.com.br

Recebido: 07/04/2013. Aceito: 28/11/2013 